\title{
Review of Technologies for Automatic Health Monitoring of Structures and Buildings
}

\author{
Artem Basko $^{1}$, Olena Ponomarova ${ }^{2}$, and Yurii Prokopchuk ${ }^{3}$ \\ 1,2,3 Prydniprovska State Academy of Civil Engineering and Architecture, Dnipro, Dnipropetrovska, 49600, Ukraine \\ basko.artem@pgasa.dp.ua \\ pricmech@ukr.net \\ itk3@ukr.net
}

\begin{abstract}
Research in the field of structural monitoring of structures, buildings and structures is not abating. A key link in a modern wireless monitoring system is a sensor built using wireless technologies. Undoubtedly, wireless sensors are gradually replacing wired systems that are difficult to maintain, connect and costly. However, we should not forget about wired systems, wireless sensor networks are a new stage in the development of structural monitoring.

The level of development of monitoring systems and wireless sensors for monitoring tasks has not yet been fully investigated for their universal application in various applications. There are also software restrictions associated with the creation and configuration of sensor networks.

The importance of using automatic monitoring systems lies in their application in smart homes as monitoring system for the condition of a building and as a human security system.

According to this study, it aims to provide a comprehensive overview of structural health monitoring over the years. In particular, this article reviewed and analyzed the main components of wireless communication, such as: hardware of smart wireless sensors, wireless protocol, network architecture, operating systems. This review also presents the scope of both test benches and real deployments of such systems.
\end{abstract}

\section{INTRODUCTION}

Structural condition monitoring (SHM) is a fairly successful method of non-destructive detection of various types of defects in buildings and structures, as well as performing both long-term and short-term monitoring in real time for their condition. In general, monitoring the state of structures consists of the following stages: monitoring an object for a

Artem Basko et al. This is an open-access article distributed under the terms of the Creative Commons Attribution 3.0 United States License, which permits unrestricted use, distribution, and reproduction in any medium, provided the original author and source are credited.

https://doi.org/10.36001/IJPHM.2021.v12i2.3015 certain time using a large number of sensors, primary processing of measured data to extract a useful signal, filtered data is analyzed to highlight features, characteristics, state of the observed object, as well as to determine the degree and the place of damage.

The main problems in structures and buildings are associated with the risk of aging of the structural characteristics, fatigue or damage to the structure, earthquakes, high winds and various dynamic loads. Monitoring these risks helps to analyze and evaluate the condition of the structure. And if necessary, apply appropriate measures to eliminate the risk of destruction. In particular, apply measures such as timely maintenance, structural control, signal and warn of evacuation, take various firefighting measures and provide rescue.

Thus, ensuring the safety of the structure, and, as a result, human life increases the demand for the use of structural monitoring systems. High-quality and safe operation as a whole depends on the timely and accurate detection of structural damage and an assessment of the degree of influence on the performance of the structure. The use of accelerometers as vibration sensors is the most used solution in current research as they have proven to be good at detecting damage that cannot be seen using visual methods.

The modern approach consists in the implementation of wireless sensor nodes in existing or newly created control systems for buildings or structures, which makes it possible to build substantially new engineering systems. The use of wireless sensors based on modern communication protocols, in comparison with wired systems, allows for flexible and easy deployment, remote control, and low maintenance costs. Unfortunately, predicting damage and deteriorating the characteristics of structures is quite problematic due to the lack of operating conditions and any information about the current state.

Thus, it is impossible to do without the use of intelligent methods, especially in the conditions of the complex structures being created. The future development of the 
development of technologies in the field of structural monitoring will be based on cognitive methods, that is, methods capable of exhibiting cognitive activity to the object of monitoring. Such approaches will make it possible to implement more automated and detailed control over complex objects that cannot be understood by humans. There is no doubt that the construction of automatic SHM systems is an integral stage in the development of modern technologies in various spheres of human life.

\section{HARDWARE PART OF SMART WIRELESS SENSORS}

A key component of modern monitoring systems is the wireless sensor node. The performance and quality of the obtained results of the monitoring and data acquisition system directly depends on the selected sensor components. Depending on the application used, the sensor node must have on board all the required peripheral interfaces, the key ones of which are ADC, SPI, UART.This section contains various technical solutions used for sensor networks, both industrial and prototypes used for academic purposes.

The analog signal processor uses two low noise 24 bit ADCs with sampling rates up to $192 \mathrm{kHz}$ (Araujo et al. 2012). Each ADC has two differential inputs, digital, anti-aliasing, and linear phase filters, an integrated high-pass filter, and digital sampling rate control. The sensors use a sampling rate of $3906.25 \mathrm{~Hz}$, and the already digitized information using the SPI interface transfers data to the PIC32 microcontroller, and using the USB bus it transfers data to the main control module. This control module is based on an ARM9 microcontroller as shown in Fig. 1, running a Linux system. The main controller is responsible for controlling the modules: data acquisition, wireless data transmission, synchronization, power supply. It also has a 2GB external SD card storage and can store measured data up to $500 \mathrm{~min}$. The power supply system uses lead batteries $24 \mathrm{~V}$ and $7 \mathrm{Ah}$ with a total weight of $5.6 \mathrm{~kg}$. This assembly allows full power operation for 15 hours.

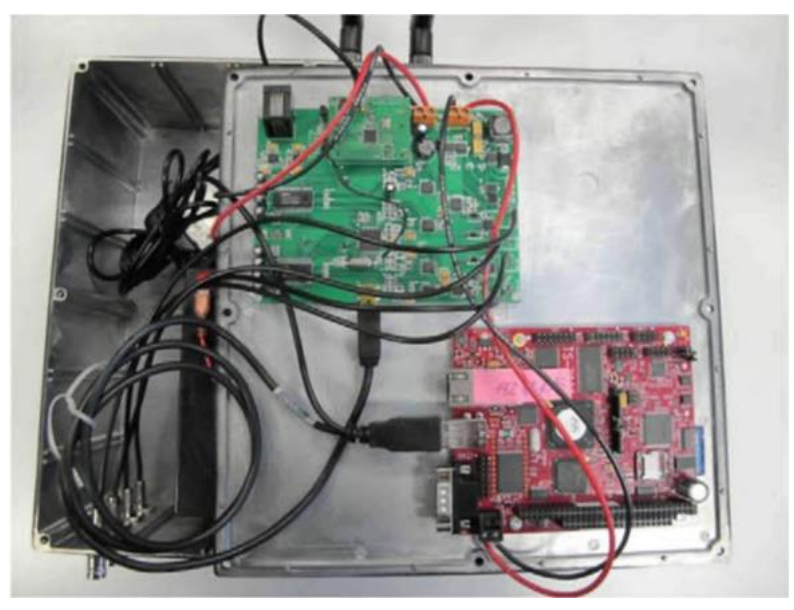

Figure 1. Wireless sensor based on PIC32 (Araujo et al. 2012)
The presented system by Pandey et al. (2016) involves the development of a flexible, inexpensive and autonomous system for monitoring the state of structures using the Internet of things. The presented sensor node is a server that hosts a website that consists of a Wi-Fi router. Uses the Arduino Uno platform on an ATMEGA 328 microcontroller it is displayed on Fig. 2. The microcontroller has 13 digital and 5 analog pins for operations, as well as peripheral interfaces such as SPI and I2C. The microcontroller is programmed using the $\mathrm{C}$ programming language via the USB port. The Adafruit Wi-Fi CC3000 is used as a Wi-Fi module, which transmits data via the SPI bus. To track vibration effects, the board has a three-axis accelerometer MMA8452Q, it is configured for acceleration +/- 8G. It has a high bandwidth up to $800 \mathrm{~Hz}$ with 12 bit precision.

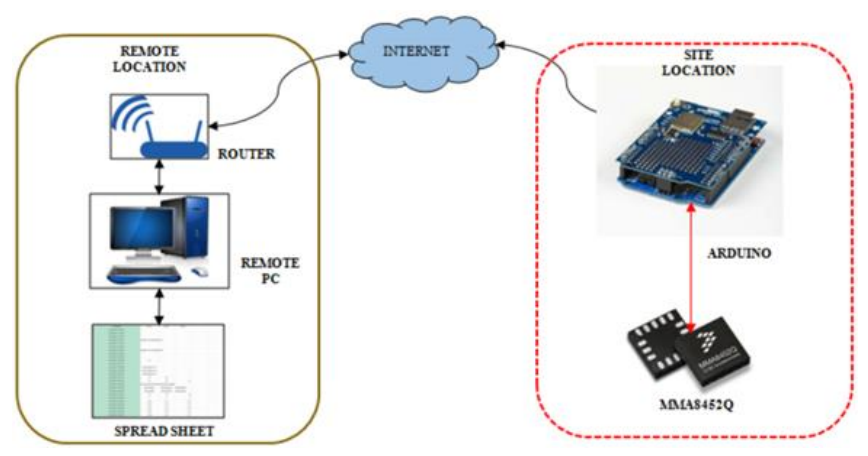

Figure 2. Sensor based on Arduino UNO (Pandey et al. 2016)

Bridge et al. (2010) presented monitoring systems built on commercial platforms the platform of smart sensors Imote2 was used, as shown in Fig. 3. Imote2 is an intelligent sensor that was built on Intel $\mathrm{X}$-scale processor, the main advantage of the platform is high scalability of processor speed. Key features such as $256 \mathrm{~KB}$ SRAM, $32 \mathrm{MB}$ flash and external SDRAM enable the processor to perform high performance computing with large data samples. Since the Imote2 architecture does not imply the presence of an analog-todigital converter (ADC), therefore, an SHM-A expansion board with a 16-bit four-channel Quickfilter QF4A512 ADC was used, which allows you to program the required sampling rate and digital filter parameters, namely FIR, with the ability to change the coefficients up to 512. The SPI protocol is used to transfer digitized data. An STMicroelectronics LIS344ALH capacitive MEMS accelerometer is used as a vibration sensor. The resulting data drift due to the heating of the Imote 2 board as a result of the work was eliminated by software, but this shows the difficulty of using it in a confined space.

Some study (Sarwar et al. 2020, Zhu et al. 2018, Fu et al. 2018, Spenceret al. 2017)are using powerful, high performance, commercial wireless sensor called Xnode it is showed in Fig.4. The sensor presented in the collection system [6] consists of several boards, each of which corresponds to a certain functionality. So the MINI4357 
board with an integrated LPC4357 microcontroller operates on two Cortex M4 / M0 cores at a configurable frequency up to $204 \mathrm{MHz}$. The microcontroller board has both standard general-purpose I / O pins and peripheral interfaces SPI, I2C.
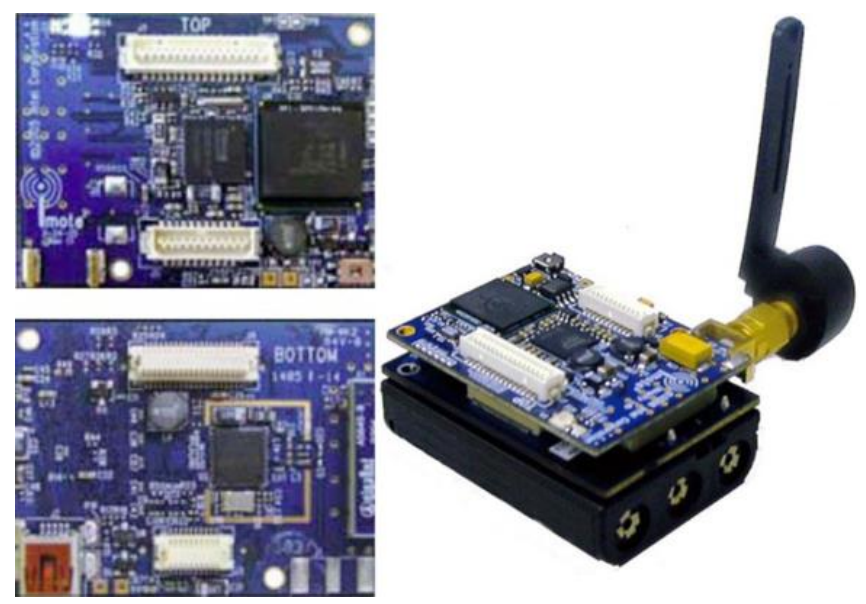

Figure 3. Commercial smart sensor Imote2 (Bridge et al. 2010)

The provision of radio frequency communication is performed on the radio receiver board and the power management board, which can control the charge from both the mains and solar panels. The radio transceiver itself is based on the Zigbee module and operates at $2.4 \mathrm{GHz}$. The sensor board has a 24-bit ADS131E08 ADC with built-in 8 channels and a sampling rate of up to $16 \mathrm{kHz}$. The board is preinstalled with a three-axis analog accelerometer LIS344ALH.

To improve the energy efficiency of the system, the wireless smart sensor is triggered and accordingly supplies power to the main boards upon event (Sarwar et al. 2020). Vibration and strain sensors generate a logic signal by accelerating at a certain predetermined threshold, which makes the controller wake up. Also, the built-in timer periodically turns on the microcontroller for periodic control of the structure.

The power supply and power consumption of the main measurement modules of the sensor were calculated to assess the operating time and energy efficiency of the system. The system uses an ADXL362 acceleration sensor to detect vibrations, it has a low power consumption of $13 \mu \mathrm{A}$ and a sampling rate of $400 \mathrm{~Hz}$. Also, the sensor has a built-in system for determining a predetermined threshold, which can serve as a wake-up signal.

A Wheatstone bridge (FLA-3-350-11-1LJC) is used to detect strain with amplification of the signal to obtain the desired response when applied to the sensor. An INA2128 precision microcircuit with a gain of up to 10,000 times is used as a signal amplifier. Timed events work on the RTC DS1342 chip. In functionality, it has the ability to save the event time and has two emergency contacts, as well as, like other microcircuits, have a low current consumption.
For logical interfacing of individual modules, a logical OR chip SN74AUP was used in order for the microcontroller to determine whether to turn on the power or not. An 8-bit Microchip ATtiny85 was used as the main microcontroller, which has all the necessary characteristics such as: SPI and $\mathrm{I} 2 \mathrm{C}$ interfaces, low power consumption.

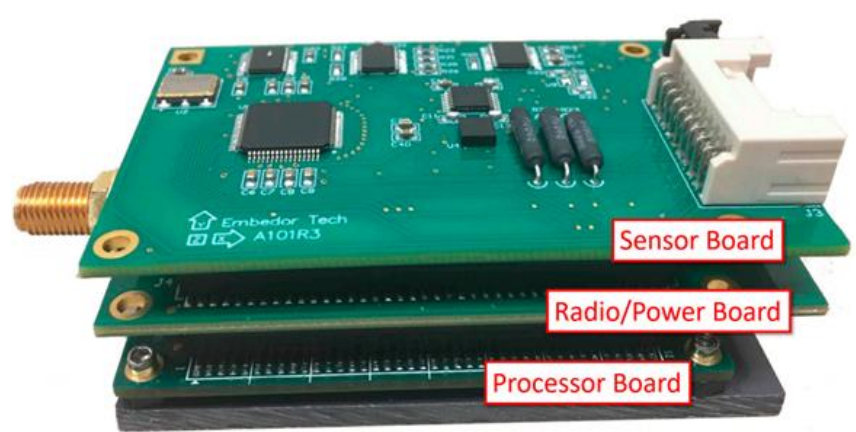

Figure 4. Xnode board (Zhu et al. 2018)

As in the previous study, the Xnode platform was used, but a distinctive feature is a detailed description of the selection of a sensitive vibration element (Zhu et al. 2018). The Epson MA351 digital high-sensitivity accelerometer has all the necessary characteristics for monitoring systems. The resolution is $0.06 \mathrm{ug} / \mathrm{LSB}$, and the acceleration measurement range is $-5 \mathrm{~g}$ to $+5 \mathrm{~g}$. The accelerometer has a built-in configurable FIR filter and the ability to program the cutoff frequency. The presence of serial interfaces SPI and UART makes it convenient for use in most devices. The real characteristics of the sensor were evaluated experimentally on high frequency vibrations and environmental vibrations.

The use of a vibration detection trigger system using the Xnode board requires a reasonable selection of the sensor ( Fu et al. 2018). The study carried out a comparative analysis of the choice of a low-power trigger accelerometer. Among the compared trigger accelerometers ADXL362, LIS3DSH, MPU6050, the ADXL362 was chosen because of its power consumption. The start to receive data is performed by an interrupt from the accelerometer, which can occur when a specified threshold is exceeded. The triggered interrupt turns on the MOS transistor, which in turn turns on the Xnode board, and the board is turned off by a low interrupt level in the same way. You can also turn on the transistor and in time for this, a DS3231 real-time clock chip is installed.

Monitoring systems based on IoT sensors use a wireless network to collect data in real time (Basyazıc1 et al. 2018). Data collection systems are based on an inexpensive ESP 8266 12E module which has on board: Wi-Fi with built-in TCP / IP protocol. The core of the module has a 32-bit architecture and operates at a frequency of up to $160 \mathrm{MHz}$. It works in tandem with a DHT22 digital temperature and moisture sensor it is shown in Fig. 5. Chang and Lin (2018) presented a 3D model for displaying the movement of a monitored object. A distinctive feature of the sensor from the previous system is the use of the ADXL345 accelerometer. 


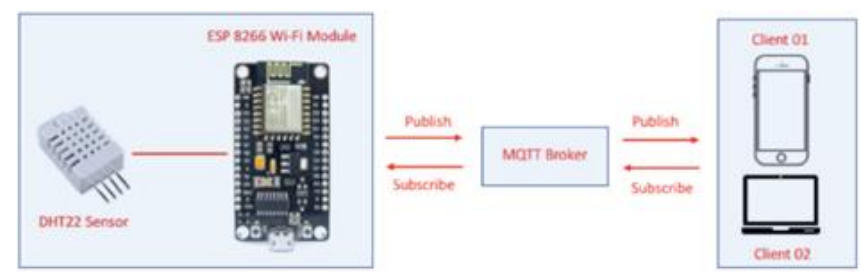

Figure 5. Wireless IoT sensor based on ESP $8266 \mathrm{Wi}-\mathrm{Fi}$ (Basyazıc1 et al. 2018)

Torfs et al. (2018) have been used a bundle of an Atmel ATZB-900 IEEE 802.15.4 compliant wireless data transmission module operating at $900 \mathrm{MHz}$ and a data acquisition module running on a TI MSP430 microcontroller together with an Analog Devices AD7683 16-bit ADC, it is shown in Fig. 6. The entire device is powered from the mains, but a Tadiran SL-2770 lithium-thionyl chloride battery is installed to ensure uninterrupted operation.

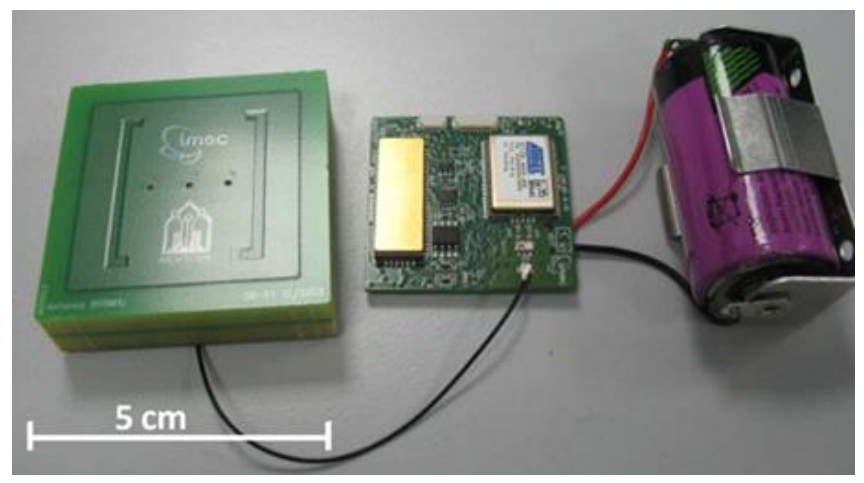

Figure 6. Low Power Wireless Sensor based on Atmel ATZB-900 (Torfs et al. 2018)

Presented research by Wang et al. (2012) has focused on the main aspects of sensor data processing such as sampling rate, data filtering and noise reduction. For example, the use of a strain and angle sensor is sampled at a frequency of $1 \mathrm{~Hz}$, and a vibration sensor at frequencies from $10 \mathrm{~Hz}$ to $1000 \mathrm{kHz}$. Thus, the imposed sampling rate requirements impose additional system overhead. At the same time, image sensors make rather high demands on the information transfer rate and image processing and decoding capabilities.

The sensor presented by Loubet et al. (2019) is built on the STMicroelectronics B-L072Z-LRWAN1 development board, which consists of an STMicroelectronics STM32L072CZ microcontroller built on an ARM Cortex M0 + core and a Semtech LoRa SX1276 transceiver. The study also compares four wireless data transmission technologies (LoRaWAN, Bluetooth Low Energy, RFID and RuBee). For a full comparison of all wireless technologies, Wi-fi and ZigBee had to be added to the list. And there is no need to consider RuBee technology at the moment, since it is not available for commercial use. All construction of the sensor prototype has shown in Fig. 7.

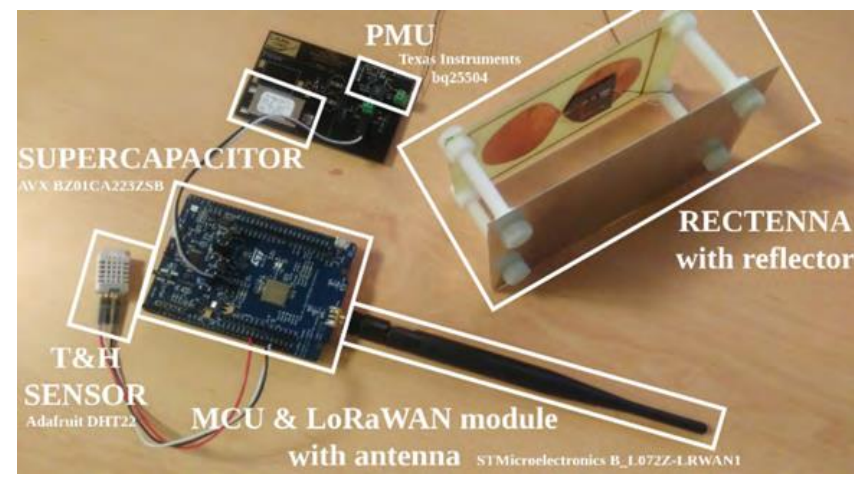

Figure 7. Sensor prototype based on LoRaWAN (Loubet et al. 2019)

The multifunctional sensor used in the system has the ability to measure and track acceleration, deformation, humidity and temperature (Somwanshi and Gawalwad 2015). In the study dIGITEXX d110-U (uniaxial) accelerometer and the OS3155 is a rugged strain gage sensor were used. Such sensors enable the deployment of a wireless smart sensor network using ZigBee protocols. Communication in the system is provided using the IEEE 802.15.4 standard and operates at speeds from $20 \mathrm{kbps}$ to $250 \mathrm{kbps}$. The IoT structure for $\mathrm{SMH}$ is built on a Raspberry Pi 2 board that works in conjunction with a Wi-Fi module (Abdelgawad and Yelamarthi 2017). The system runs under the Linux operating system on an ARM Cortex-A7 processor, which provides a fairly large computing power. A high-speed CMOS ADC CA3306 is used to operate the analog signal, and the $74 \mathrm{HC} 4050$ is used as a voltage level converter.

The hardware part of the data collection system is of interest since it is built on a ZigBee wireless communication module and a GSM communication module, which provides remote monitoring and control (Kareem and Al-Sulaifanie 2017). The main core of the node is the Atmega328P microcontroller with a built-in ADC that operates at a frequency of $9.6 \mathrm{kHz}$. The node also consists of a power module, a microcontroller, sensors for measuring power, voltage, current, temperature. Digitized data of voltage, current, temperature, power is sent to the central node. The operation of the node is indicated by the block diagram has shown in Fig. 8 in the article.

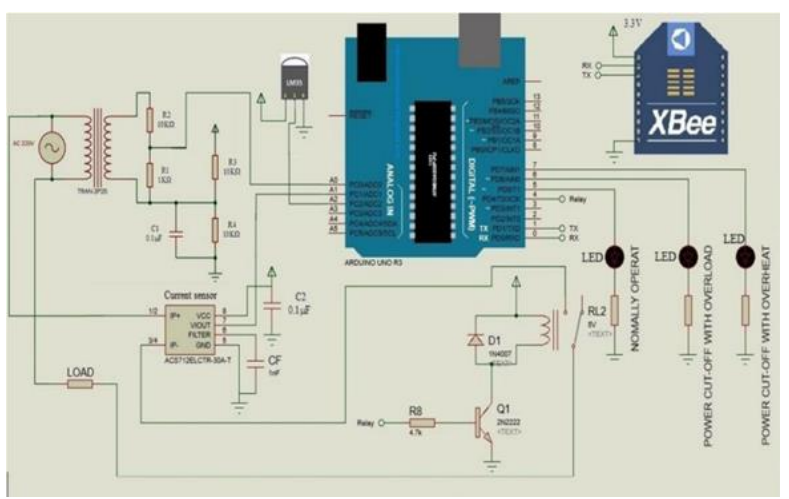

Figure 8. Sensor prototype based on Arduino Uno and Xbee (Kareem and Al-Sulaifanie 2017) 
The monitoring system is triggered by a trigger and begins to process the sensor data, after conversion, the data is transmitted to the wireless module (Hailing et al. 2019). The system used an STM32L476ZE microcontroller from STMicroelectronics running on a 32-bit ARM® Cortex ${ }^{\circledR}-\mathrm{M} 4$ core that has a 24-channel ADC. To ensure noise suppression of the environment and the turbojet, a high-pass filter of the 2nd order with a cutoff frequency of $2.1 \mathrm{kHz}$ was applied. The wireless module "Digi XBee® Zigbee" is used for data transmission. The structure of wireless data transmission is shown in Fig.9. When an event occurs, the node sends a data packet from the sensors to the station, and the station, upon receiving the message, sends a response to the node about successful data transfer and the node goes into sleep mode.

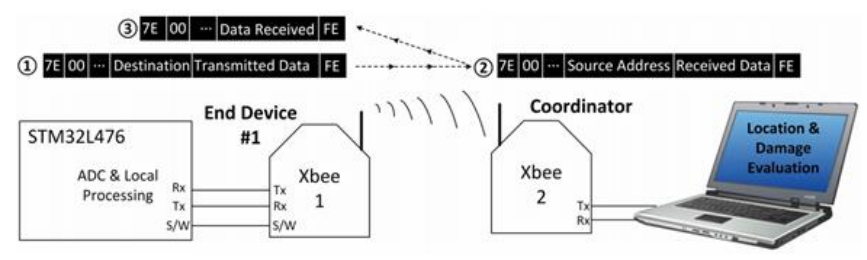

Figure 9. Structure of wireless data transmission (Hailing et al. 2019)

The presented articles showed that there is no single universal already made solution. It depends on the specific application, all main components are selected, such as an ADC (its resolution, conversion speed), a microcontroller, a data transmission module, power supply, and also types of sensors.

Unfortunately, many of the presented systems have excess computing power, which is not used in the first place, since the data is not processed on the microcontroller, but transmitted to the main server, which begins to perform operations on the received data from the sensors. Secondly, they have a fairly high cost, which is impractical when using sensor networks based on multiple sensors. If you use networks based on ESP 8266 12E modules, they are inexpensive compared to commercial solutions, but they do not provide the necessary transmission range in comparison of the system with Zigbee modules. Also, the separation of each component separately increases the cost of the overall sensor design.

Very few scientists pay attention to power management. And such solutions are difficult to use since they do not have a battery recharging system, which requires timely replacement of batteries and are also quite cumbersome. Based on the fact that wireless sensors are installed close to the outside of the building, small solar panels could be used for recharging, which would make the sensor nodes autonomous for extended periods of use.

\section{Wireless COMMUNICATION PROTOCOL}

The development of a wireless sensor network including wireless sensors depends on the industry of application, which is associated with the selection and use of a specific wireless technology. To solve the problems of monitoring the state of structures and buildings, such technologies as IEEE 802.11 Wi-Fi, IEEE 802.15.1 Bluetooth, IEEE 802.15.4 ZigBee are mainly used.

Most of the monitoring systems are built on the IEEE 802.15.4 standard using the Zigbee data transfer protocol. Zigbee modules operate at $868 \mathrm{MHz}, 918 \mathrm{MHz}$ and $2.4 \mathrm{GHz}$, which provides fast and noise-free communication between nodes in the structure (Sarwar et al. 2020, Zhu et al. 2018, Fu et al. 2018, Spencer et al. 2017, Torfs et al. 2013, Abdala and Salih 2012, Wang et al. 2012). This standard is widely used in such areas as scientific, medical, industrial etc.

The analysis of scientific works showed that, in general, the $\mathrm{Wi}-\mathrm{Fi}$ protocol in monitoring systems is used to transfer data directly to the data processing server from the main node. Only a few works are devoted to the construction of a full sensor network using Wi-Fi technology. They have such disadvantages as low range and high power consumption.

The Wi-Fi used in the study sends all data packets with high bandwidth (Araujo et al. 2012). The server creates a Wi-Fi access point to which all nodes are connected, and data is exchanged using a secure SSH protocol, which allows you to access data using a special security key, while the transfer rate averages 1.3 $\mathrm{MB} / \mathrm{s}$. To avoid collisions during data transmission, the server sends commands to request data, and can also retransmit packets in order to recover lost data. The received data in the form of binary numbers is decoded and generates text files in ASCII format.

The sensor, which includes an Adafruit Wi-Fi module, establishes an 802.11 connection with a Wi-Fi network using a dynamic IP address using a dynamic node configuration and then starts communicating over the TCP / IP interface (Pandey et al. 2016). The microcontroller processes data from the accelerometer and generates command messages in JSON format. Further, the received data goes into a Google Spreadsheet where the user can already work with this data.

The author proposed to use the Synapse RF300PC radio frequency module, and also described the requirements for the selection of the element base of the sensor, software implementation, and the choice of the deployment structure for a wireless sensor network (Magdy and Osama 2014).

The experience of wired monitoring systems showed their main disadvantages, among which: the inability to deploy the system on ready-made buildings, the complexity of deployment during the construction process due to the large number of wires and the time for their installation, which requires the use of highly qualified specialists. Thus, the use 
of monitoring systems based on wireless sensors does not have such disadvantages that are inherent in wired systems.

\section{NETWORK ARCHITECTURE}

To select the optimal architecture of the sensor network, it is necessary to consider such factors as: the type of object in which it will be embedded, the number of main nodes, the location of nodes on the object, the construction hierarchy. The hierarchy of construction is chosen depending on the required data transfer rate over the network and the number of local sensors, which affects the amount of information per node, network fault tolerance, and self-healing.

As can be seen in Fig. 10, the network presented in the study uses a standard star topology (Torfs et al. 2013). In this network, nodes are divided into two types with different types of sensors - these are strain gauges for measuring vertical loads and three-axis accelerometers for measuring horizontal accelerations. The received data from the sensors using a wireless communication channel transmit data to the base station via a direct antenna. The base station processes and transfers data to the Internet.

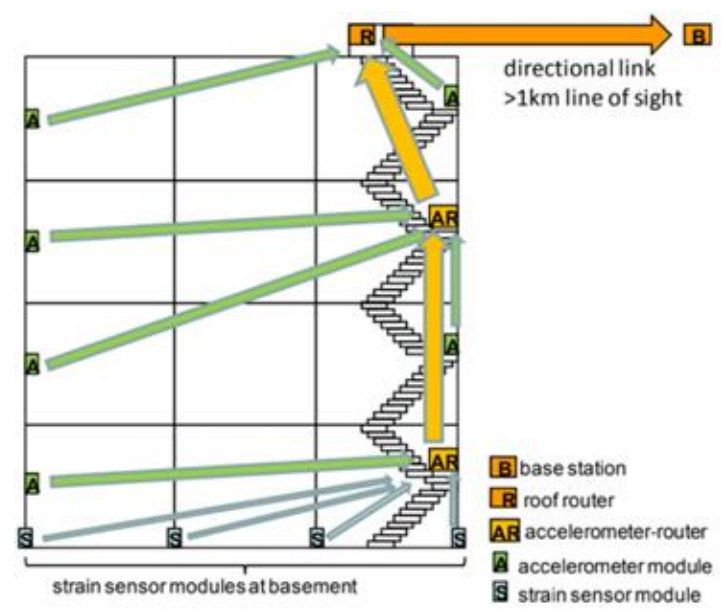

Figure 10. Sensor network topology for building monitoring (Torfs et al. 2013)

In the articles presented by authors (Abdala and Salih et al. 2012, Wang et al. 2012), the application of a sensor network using the ZigBee standard based on IEEE 802.15.4 allows the deployment of various network topologies, including star, mesh and tree, which is shown in Fig. 11.

The study reveals the characteristics of each of these topologies, their main advantages and disadvantages. The built system based on ZigBee modules has such advantages as: increased deployment flexibility, cost-effectiveness, energy efficiency, self-healing network, sufficient network bandwidth.

Based on the initial concept, the sensors should transmit data to the server, so a star topology was chosen for the monitoring system (Loubet et al. 2019, Somwanshi and Gawalwad
2015). The sensors track and react to such phenomena as earthquakes, strong winds, dynamic loads. In this system, sensors do not have the ability to send data on their own, but can only send when a request is received from the server. This approach prevents data collisions.

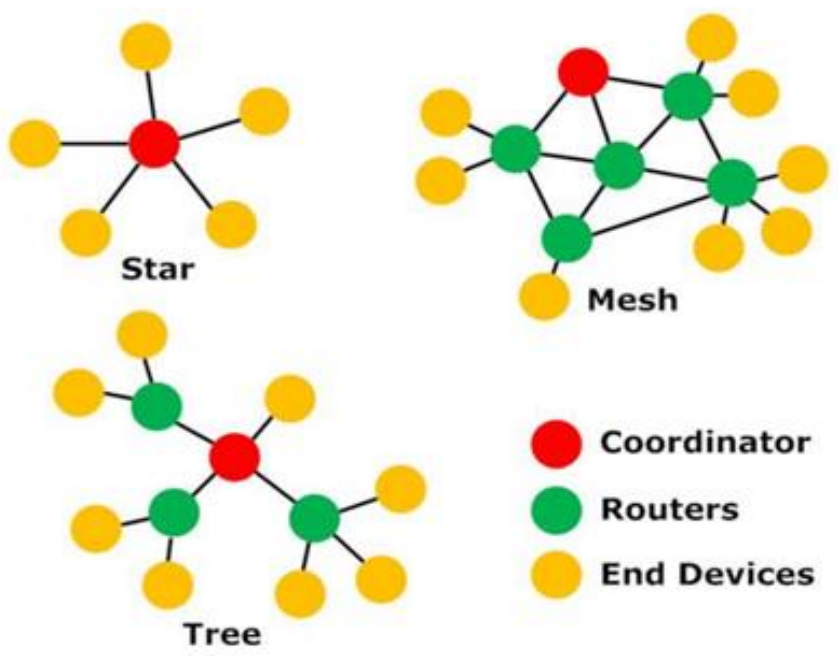

Figure 11. Different types of sensor network topology

(Torfs et al. 2013)

A new approach deserves special attention, namely, consideration of the problem of constructing cognitive architecture for the purpose of monitoring buildings and structures (Serov 2017). The use of cognitive technologies in monitoring is the next stage in the development of technologies in this area. The author assumes the use of a dynamic artificial neural network, the main essence of which is the appearance of a new neuron or a connection between existing neurons when new knowledge about the system arises.

Structurally, the proposed neural network has several layers of neurons: input, intermediate and output layers. The input layer neurons are a kind of filter set. Intermediate layers perform a spatio-temporal representation of the dynamics of the object under study. The output layer is the output layer that the neural network receives from observations.

Thus, there are many factors in choosing the hierarchy of the sensor network. In general, the structure of the sensor network is limited by the communication standard itself, the physical module that implements the wireless communication, and software implementation.

The main disadvantage of modern monitoring systems is the presence of complex scientific and technological structures, which requires large economic and time costs. And also, the availability of qualified personnel to correctly assess the results obtained. Therefore, the development of new automatic self-learning cognitive systems is a priority area of research. 


\section{OPERATING SYSTEM}

Applications use either operating systems or embedded software to interconnect hardware components. The vast majority of existing commercial sensors use a variety of operating systems. Depending on the field of application of the monitoring system, the sensor nodes used and the hardware used, the application of various operating systems also varies.

The Imote2 smart sensor platform uses the popular TinyOS operating system (Bridge et al. 2010, Spencer et al. 2017). The structure of program execution in the operating system is divided into two main types: execution of tasks and processing of hardware events. Such parallelization of tasks makes programming and debugging a rather complicated process, since it requires the description of a large number of subroutines and event handlers, in particular, network functions and data processing algorithms. Based on this, the ability to control the execution of a task in real time is absent, since any process can interrupt each other.

Basyazıc1 et al. (2018) uses the MQTT data transfer protocol in place with the TCP / IP protocol using the standard port 1883. The Arduino IDE and the $\mathrm{C}++$ programming language were used for software development. The software implements such functions as: energy saving, interval measurements, dynamic response thresholds. In this structure, sensors have the ability to directly connect to the network, so a specific connection topology is not required.

The transmitted data from the sensors use a Wi-Fi wireless network (Chang and Lin 2018). The processing of the received data is performed using cloud computing on the Internet. The presented system uses two different servers, one of which is engaged in computing and storing processed data, and the second serves as an intermediate server for real-time data exchange with sensors. The servers are deployed on the Ubuntu operating system. Data from the accelerometer comes with a sampling rate of $20 \mathrm{~Hz}$, they are also buffered on board the sensor, and then sent to the server where the processed data is stored in the MongoDB database.

The studies that use the Xnode board run on the FreeRTOS operating system (Sarwar et al. 2020, Zhu et al. 2018, Fu et al. 2018, Spencer et al. 2017). The software used SOA-based Illinois SHM Services Toolsuite tools. The main loop is subdivided into three main sub-tasks with different levels of priority. The main function is processing and collecting data as this is the most important process. In second place is the task of radio frequency communication, which ensures the efficiency of data transmission. To implement the software, the $\mathrm{C}$ programming language was used, which simplifies the development process and the use of third-party data processing libraries.

Thus, the sensors use both various operating systems and embedded software. By its nature, the sensor node is a builtin hardware node in the monitoring system. Therefore, it is necessary to develop a node on the basis of embedded software using various programming languages $\mathrm{C}$ and $\mathrm{C}++$. Embedded software development undoubtedly increases the reliability and safety of the software since the logic of operation and various functionalities are hidden from the environment and are known only to the developer. At the same time, sensor nodes built on various operating systems have many vulnerabilities for hacking, since they are open source.

\section{SPHERE OF APPLICATION}

Wireless systems for monitoring the state of structures and buildings by a fairly new method have not gained widespread use during research and development. Most of the research is more on research capabilities in search of optimal regimen and to a lesser extent on practical action. Therefore, many experiments were created in the laboratory with the aim of creating a prototype device. And only a coalition of some is aimed at the practical activities of scientists. All of this suggests that technology continues to be used by many unresolved issues to preserve research for scientists.

Depending on the object of monitoring, such systems help to identify and assess the impact of various phenomena on the state of the structure of the object of monitoring. So, in the general case, among the natural phenomena, one can distinguish strong gusts of wind, tornadoes, icing, mudflows, sinkholes in the ground, earthquakes. Also important is the influence of artificial disturbances near the monitoring object when drilling shafts for piles, constructing a new building or underground metro, if damaged by another artificial object.

In the applied finite element calculation method and a real experiment was carried out on the Spanish Bridge in the village of Magaz de Pisuerga is depicted in Fig. 12. The vibration of the bridge was detected at 90 planned nodes. The results obtained were compared with the finite element method and showed a fairly similar result (Araujo et al. 2012).

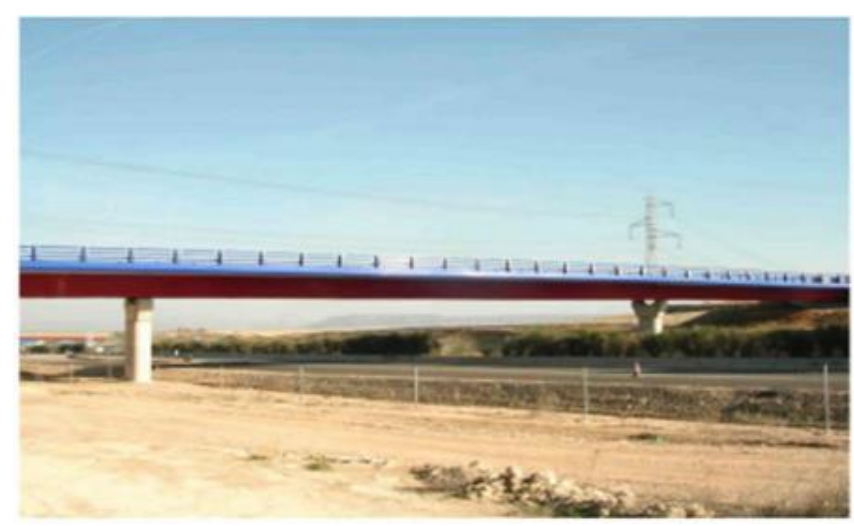

Figure 12. Magaz de Pisuerga Bridge (Araujo et al. 2012)

Bridge et al. (2010) in a study, the monitoring system used was tested on the cable-stayed bridge the 2nd Jindo Bridge in 
South Korea, as depicted in Fig. 13. The main goal of deploying smart sensors is to try and implement efficient applications. During the deployment of the system, 70 Imote 2 nodes were installed, during the operation period of 4 months, the main problem of the system deployment was the optimization of the software parameters.

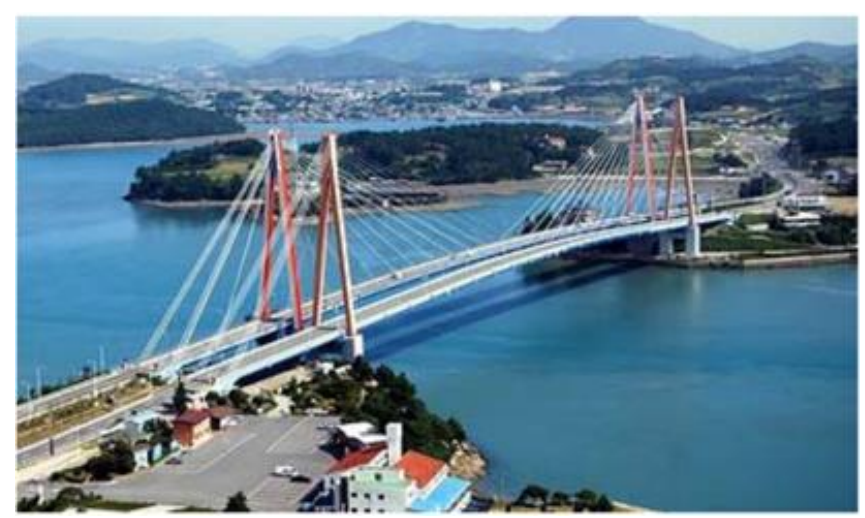

Figure 13. Different types of sensor network topology

(Bridge et al. 2010)

The constructed wireless sensor by Zu et al. (2018) was tested experimentally on a vibration table and in the field. A vibrating table with 6-DOF degrees of freedom made it possible to evaluate the measured efficiency of the selected accelerometer, it has shown in Fig. 14. The experiments carried out showed a high similarity between the results of the reference sensor and the tested one, which makes it possible to accurately measure the disturbing vibration.

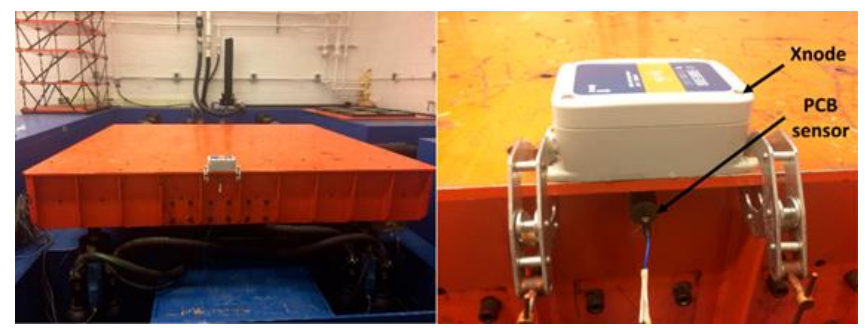

Figure 14. Xnode sensor on vibration table ( $\mathrm{Zu}$ et al. 2018)

To implement the presented monitoring system, tests were carried out on a railroad bridge in Fig. 15 in Illinois (Fu et al. 2018). The sensor successfully responded to the passage of the train, which is quite similar to sudden emerging phenomena such as natural, artificial. As an example of natural phenomena are: strong winds, earthquakes, sinkholes, mudflows, cave or snow storms. Among the artificial phenomena, the following can be distinguished: mechanical impact as a result of damage by another artificial object, the effect created as a result of the process of construction of nearby buildings or underground metro stations. Carrying out a comparative experiment of wireless and wired sensors, showed that the results obtained by the author made it possible to assess the condition of the bridge.

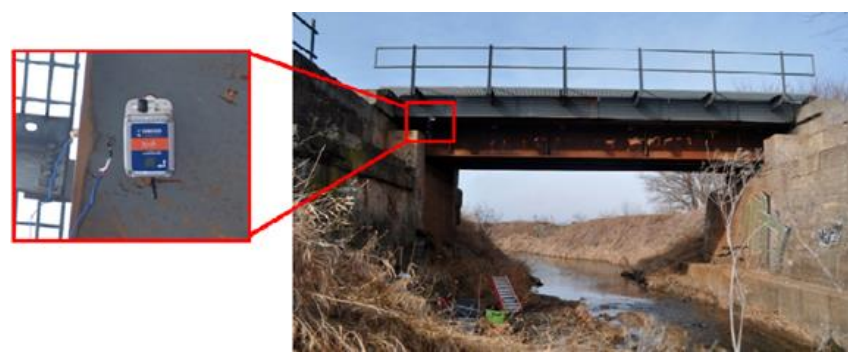

Figure 15. Testing a wireless sensor network on a railway bridge (Fu et al. 2018)

Pandeyet al. (2016) presented a laboratory experiment using cracked and intact beams as it is shown in Fig. 16. The vibrations in three directions were read by an accelerometer for each type of beam. A lot of data was obtained from vibration response and after that have been processed by computer. The application of the fast Fourier transform made it possible to obtain the intrinsic frequency response of the beam cross section that equal $52.81 \mathrm{~Hz}$.

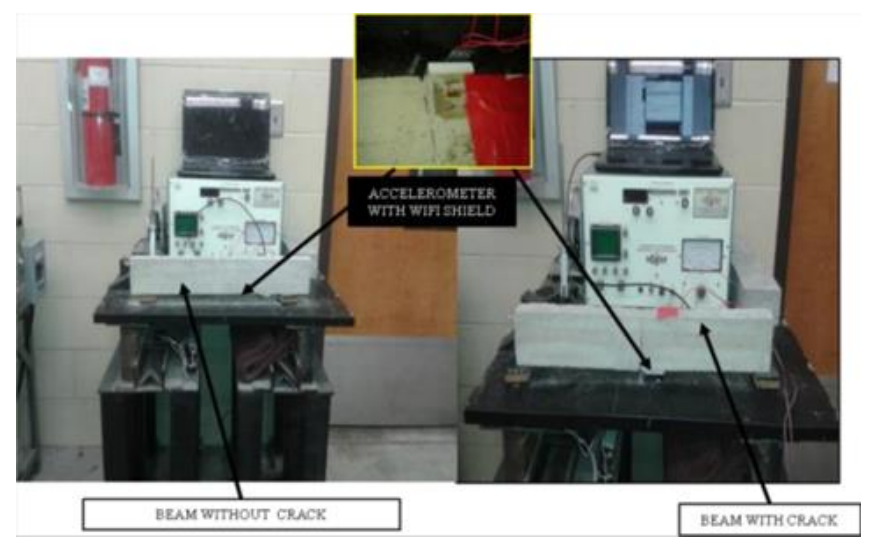

Figure 16. Sensor test bench (Pandey et al. 2016)

Sarwar et al. (2020) laboratory experimental tests with an aluminum cantilever girder and a FLA-3-350-11-1LJC strain gauge. An Xnode module with an accelerometer mounted on the top of the test bench, a strain gauge built into the bottom as shown in Fig. 17.

The article describes possible problems and solutions when building a wireless sensor network for monitoring multiple parameters (Wang et al. 2012). The use of the ZigBee standard using different topologies was aimed at monitoring power electronics, generators, wind turbine blades, and gearboxes for the FP7 Project "Monitoring the health of offshore wind farms" that shown in Fig. 18.

The unique application of the sensor network for the aviation industry has been described in a study (Hailing et al. 2019). The designed system is required to detect impacts in composite aircraft as shown in Fig. 19. The main modules were developed: data filtering, data processing and identification by trigger. The system has shown high measurement accuracy and resistance to ambient noise. 


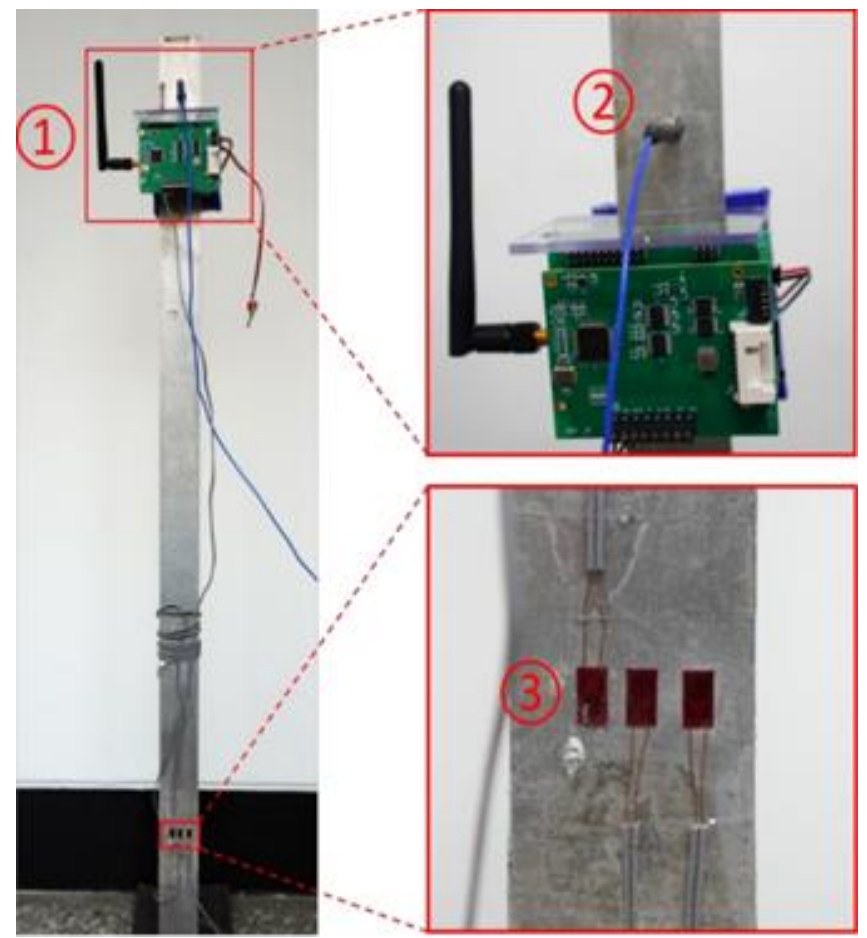

Figure 17. Experimental setup with Xnode sensor (Pandey et al. 2016)

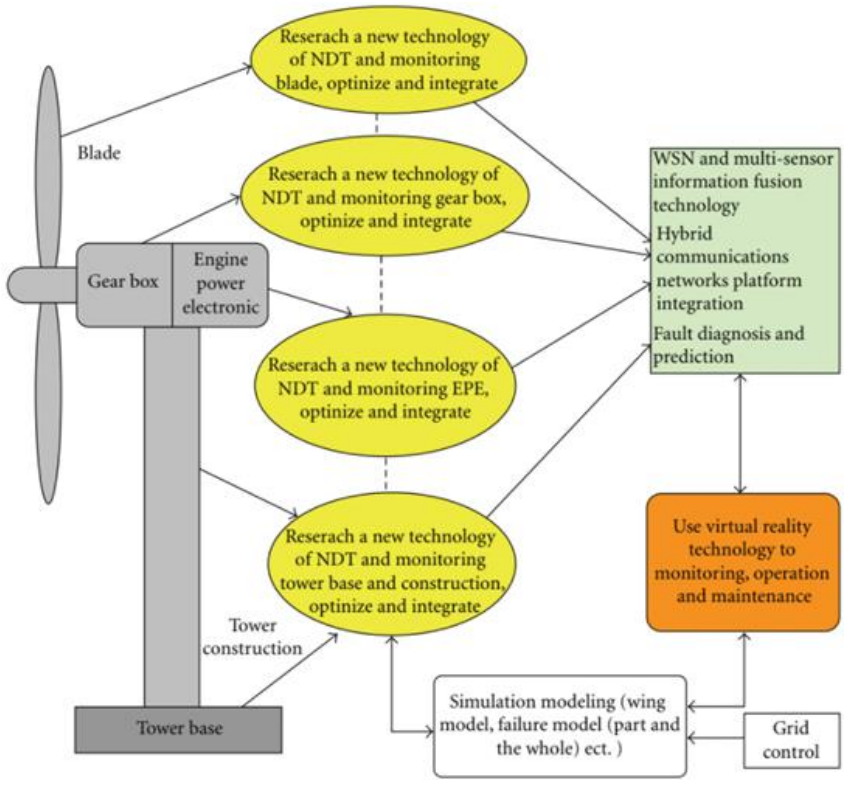

Figure 18. The structure of monitoring the parameters of a wind farm (Wang et al. 2012)

For each type of structure, the characteristics for installing the sensor network will differ, see Table 1 . This will be directly influenced by next factors: the data transmission standard, the type of sensor placement (internal or external mounting into structure), and noisy vibration environment. To reach stable signal between wireless sensors it is necessary to place them at a certain distance. In common for sensors mounted inside the structure, requires average distance between sensors which is 8 meters or closer. But for sensors with external installation, the placement distance can be increased up to 30 meters.

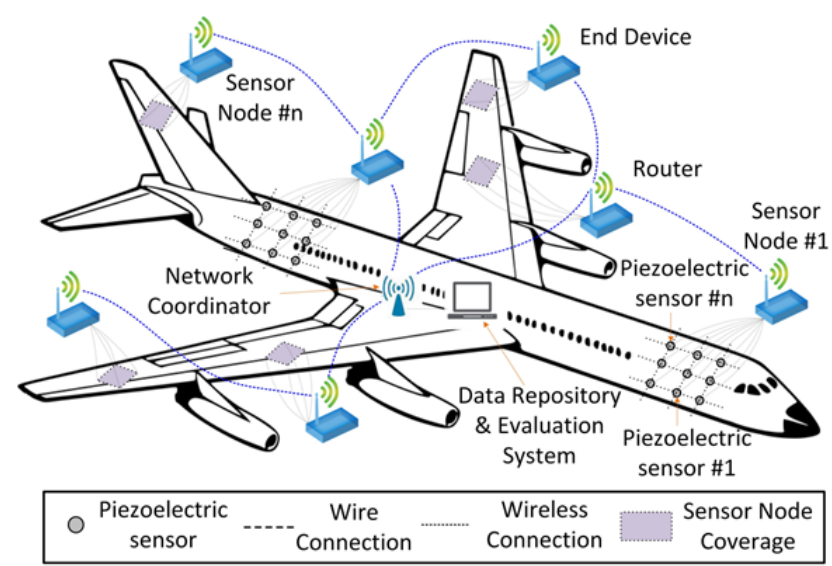

Figure 19. Location of the sensor network on the plane (Hailing et al. 2019)

The aggressive vibration character implies the presence of additional external vibration (in the case of road bridges, vibration is constantly created by the movement of cars, it can be also influenced by rivers or seas). Such conditions lead to select sufficiently sensitive sensors and accordingly process the data in the software.

\begin{tabular}{|c|c|c|c|}
\hline $\begin{array}{c}\text { Construction } \\
\text { Type }\end{array}$ & $\begin{array}{c}\text { Mount } \\
\text { type }\end{array}$ & $\begin{array}{c}\text { Vibration } \\
\text { character }\end{array}$ & $\begin{array}{c}\text { Number of } \\
\text { sensors, pcs }\end{array}$ \\
\hline Bridge & External & Aggressive & $50-100$ \\
\hline Railway Bridge & External & Normal & $30-60$ \\
\hline Building & Internal & Normal & $80-160$ \\
\hline Wind turbine & Internal & Aggressive & $10-30$ \\
\hline Plane & Internal & Aggressive & $15-40$ \\
\hline
\end{tabular}

Table 1. Summary characteristics to install a sensor network.

Thus, wireless sensor networks find applications in a wide range of both science and industry. In addition, each application requires its own individual approach in the choice of sensors, wireless standard, and sensor network topology.

\section{CONCLUSIONS AND FUTURE WORKS}

The modern approach to using wireless sensors has become dominant over wired systems and has practically replaced them and showed its advantages.

Since the most important object for a wireless sensor network is the sensor node, all efforts and attempts of scientists are aimed at creating a universal solution and improving existing methods, algorithms, hierarchies, hardware and software. The analysis showed that most of the solutions are developed superficially and do not give a complete picture for use in real operating conditions. 
As before, an important problem in WSN is the creation on the one hand of inexpensive but at the same time sufficiently accurate and sensitive sensor nodes. From the software side, the problem is to develop a large-scale network with algorithms to ensure reliable communication in a fairly noisy environment.

The use of the IEEE 802.15.4 standard has shown itself to be on the good side, since it has low power consumption and a sufficient range for data transmission. The ability to use different network hierarchies makes it the preferred standard for use in sensor nodes.

Among the main disadvantages of modern sensor monitoring systems are: the cost of a sensor unit, inefficient use of computing power, the need to have qualified specialists to analyze the data obtained.

Powering wireless sensors also requires some attention as it is necessary to use energy efficient circuitry and software solutions to ensure continuous operation of the sensors.

Further stages of development will be aimed at reducing the cost of the sensor node by selecting a cheap controller, with many built-in peripheral modules, cheap sensors and increasing the fault tolerance of both the sensor node and the system in general. The introduction of cognitive, selflearning sensor networks will make it possible to build completely self-sufficient devices, thereby excluding specialists who are engaged in data analysis. The development of a new sensor node must be performed on an embedded system without using operating systems, since any operating system is susceptible to unauthorized access to the system, which reduces the security of the system.

\section{REFERENCES}

Araujo, A., García-Palacios, J., Blesa, J., Tirado-Andrés, F., Romero, E., Samartín, A. \& Nieto-Taladriz, O. (2012). Wireless measurement system for structural health monitoring with high time-synchronization accuracy. IEEE Transactions on Instrumentation and Measurement, 61(3), 801-810. https://www.doi.org/10.1109/TIM.2011.2170889

Pandey, S., Haider, M. \& Uddin, N. (2016). Design and implementation of a low-cost wireless platform for remote bridge health monitoring. International Journal of Emerging Technology and Advanced Engineering, 6(6), 57-62.

Bridge, J., Mechitov, K., Sim, S., Nagayama, T., Jang, S., Kim, R., Spencer, B., Agha, G. \& Fujino, Y. (2010). Flexible smart sensor framework for autonomous structural health monitoring. Smart Structures and Systems, 6(5), 423-438. https://www.doi.org/10.12989/sss.2010.6.5_6.423

Basyazıc1, U., Fenercioğlu, A. \& Uhla, U. (2018). Continuous commissioning and monitoring buildings with IoT sensor networks. 13th International HVAC+R
\& Sanitary Technology Symposium, Istanbul, Turkey, April.

Chang, H. \& Lin, T. (2018). Real-time structural health monitoring system using internet of things and cloud computing. Proceedings of the 11th National Conference in Earthquake Engineering, Los Angeles, USA, June.

Sarwar, M., Saleem, M.R., Park, J., Moon, D.S. \& Kim, D.J. (2020). Multimetric event-driven system for long-term wireless sensor operation for SHM applications. IEEE Sensors Journal, 20(10), 5350-5359. https://www.doi.org/10.1109/JSEN.2020.2970710

Zhu, L., Fu, Y., Chow, R., Spencer, B., Park, J. \& Mechitov, K. (2018). Development of a high-sensitivity wireless accelerometer for structural health monitoring. Sensors, 18(1), 1-16. https://www.doi.org/10.3390/s18010262

Fu, Y., Hoang, T., Mechitov, K., Kim, J., Zhang, D. \& Spencer, B. (2018). Sudden event monitoring of civil infrastructure using demand-based wireless smart sensors. Sensors, 18(12), 1-17. https://www.doi.org/10.3390/s18124480

Spencer, B., Park, J.W., Mechitov, K.A, Jo, H. \& Agha, G. (2017). Next generation wireless smart sensors toward sustainable civil infrastructure. Procedia Engineering, 171(1), 5-13. https://www.doi.org/10.1016/j.proeng.2017.01.304

Torfs, T., Sterken, T., Brebels, S., Santana, J., Hoven, R., Spiering, V., Bertsch, N., Trapani,D. \& Zonta, D. (2013). Low power wireless sensor network for building monitoring. IEEE Sensors Journal, 13(3), 909-915. https://www.doi.org/10.1109/JSEN.2012.2218680

Abdala, M. \& Salih, A. (2012). Design and performance analysis of building monitoring system with wireless sensor networks. Iraqi Journal of Science, 53(4), 10971102

Wang, P., Yan, Y., Gui, Y.T., Bouzid, O. \& Ding, Z. (2012). Investigation of wireless sensor networks for structural health monitoring. Journal of Sensors, 2012(1), 1-7. https://www.doi.org/10.1155/2012/156329

Loubet, G., Takacs, A. \& Dragomirescu, D. (2019). Implementation of a battery-free wireless sensor for cyber-physical systems dedicated to structural health monitoring applications. IEEE Access, 7(1), 2467924690. https://www.doi.org/10.1109/ACCESS.2019.2900161

Somwanshi, S.S. \& Gawalwad, B.G. (2015). Monitoring civil structures with a smart wireless sensor network. IJEAS, 2(3), 34-39.

Magdy, I. \& Osama, M. (2014). Wireless sensor networks configurations for applications in construction. Procedia Engineering, 85(1), 260-273. https://www.doi.org/10.1016/j.proeng.2014.10.551

Abdelgawad, A. \& Yelamarthi, K. (2017). Internet of things (IoT) platform for structure health monitoring. Wireless Communications and Mobile Computing, 2017(1), 1-10. https://www.doi.org/10.1155/2017/6560797 
Kareem, O. \& Al-Sulaifanie, A. (2017). Real-time power measurements in smart building monitoring system. The Journal of The University of Duhok, 20(1), 142-152. https://www.doi.org/10.26682/sjuod.2017.20.1.13

Cheng, S., Rongtao, W. \& Yanling, Z. (2018). Real-time power measurements in smart building monitoring system. IOP Conf. Ser.: Mater. Sci. Eng., 366(1), 1-7. https://www.doi.org/10.1088/1757-899X/366/1/012084

Hailing, F., Khodaei Z.S., \& Ferri Aliabadi, M.H. (2019). An event-triggered energy-efficient wireless structural health monitoring system for impact detection in composite airframes. IEEE Internet of Things Journal, 6(1), 1183-1192. https://www.doi.org/10.1109/JIOT.2018.2867722

Serov, A. (2017). Cognitive sensor technology for structural health monitoring. Procedia Structural Integrity, 5(1), 1160-1167.

https://www.doi.org/10.1016/j.prostr.2017.07.027 